\title{
RTDF2008-74020
}

\section{A DYNAMIC TEST OF A COLLISION POST OF A STATE-OF-THE-ART END FRAME DESIGN}

\author{
Michelle Priante \\ Patricia Llana \\ Karina Jacobsen \\ David Tyrell \\ Benjamin Perlman \\ US Department of Transportation \\ Volpe Center \\ Cambridge, MA
}

\section{ABSTRACT}

In support of the Federal Railroad Administration's (FRA) Railroad Equipment Safety Program, a full-scale dynamic test of a collision post of a state-of-the-art (SOA) end frame was conducted on April 16, 2008. The purpose of the test was to evaluate the dynamic method for demonstrating energy absorption and graceful deformation of a collision post.

The post aims to protect the operators and passengers in the event of a collision where only the superstructure, not the underframe, is loaded. Methods for improving the performance of collision and corner posts were prompted by accidents such as the fatal collision in Portage, Indiana in 1998, where a coil of steel sheet metal penetrated the cab car through the collision post.

The improvements made for the SOA end frame structure include more substantial corner and collision posts, robust post connections to the buffer beam and anti-telescoping (AT) beam, and corner and collision posts integrated with a shelf and bulkhead sheet. Full length side sills improved support for the end frame. This test focused on one collision post because of its critical position in protecting the operator and passengers in an impact with an object at a grade-crossing.

For the test, a 14,000-lb cart impacted a standing cab car at a speed of $18.7 \mathrm{mph}$. The cart had a rigid coil shape mounted on the leading end that concentrated the impact load on the collision post. The requirements for protecting the operator's space state that there will be no more than 10 inches of longitudinal crush and none of the attachments of any of the structural members separate.
During the test, the collision post deformed approximately 7.4 inches and absorbed approximately 138,000 ft-lb of energy. The attachment between the post and the AT beam remained intact. The connection between the post and the buffer beam did not completely separate, however the forward flange and both side webs fractured. The post itself did not completely fail. There was material failure in the back and the sides of the post at the impact location. Overall, the end frame was successful in absorbing energy and preserving space for the operators and the passengers.

\section{INTRODUCTION}

As a result of consideration of both a notice of proposed rulemaking (NPRM) for improved cab car multiple unit (MU) locomotive equipment and the accepted industry standard, a series of tests were planned to demonstrate examples of conducting such tests to shows equivalence between testing protocols. Three test scenarios are taken from the FRA's proposed rule [1]. This paper covers the dynamic test of a collision post. There are two quasi-static tests also being planned. The quasi-statics tests are on the collision post and the corner post.

Both the proposed rule and the industry standard improve crashworthiness performance due to increased static load requirements, as well as minimum energy absorption and maximum allowable intrusion into the cab. By encouraging improved energy absorption capabilities for the end frames, the rule aims to improve survivability for operators and passengers at higher collision speeds. 


\section{BACKGROUND: COLLISION INVESTIGATIONS}

Collision Loading Scenarios Full-scale crashworthiness research has focused on two generic loading scenarios. In one scenario, a leading cab car hits another train head on. In this scenario both the underframe and the superstructure of the cab car are loaded.

In a second scenario, a leading cab car hits an object at a grade crossing in such a manner that the superstructure is loaded, but the underframe is not. Since the underframe of a railcar provides the greatest strength to protect passengers, this scenario can be dangerous for operators and passengers if the end frame is breached by the object.

The series of end frame tests discussed in this paper focus on demonstrating the performance of end frame structures for an impact scenario similar to a grade crossing collision. Two collisions are described which highlight the need for improving end frame requirements for cab cars and MUs.

Collision in Portage, Indiana On June 18, 1998, a cab carled, two-car MU commuter train collided with a highway truck at a grade crossing [2]. The highway truck consisted of a tractor with two trailers. The trailers were loaded with coils of sheet steel. The second trailer, the one furthest from the tractor, was stopped on the tracks. The train collided with the second trailer, and during the impact, a coil of steel broke free and punctured the end of the car. The steel coil rolled down half the length of the car and killed three people [3].

Figure 1 shows the exterior and the interior of the cab car after the collision. The picture on the right shows the interior of the car. The collision post had been severed and pushed back into the car. Figure 2 shows the 6-foot diameter, 19-ton steel coil. This grade crossing collision is significant because the coil loaded the superstructure of the car, not the underframe.
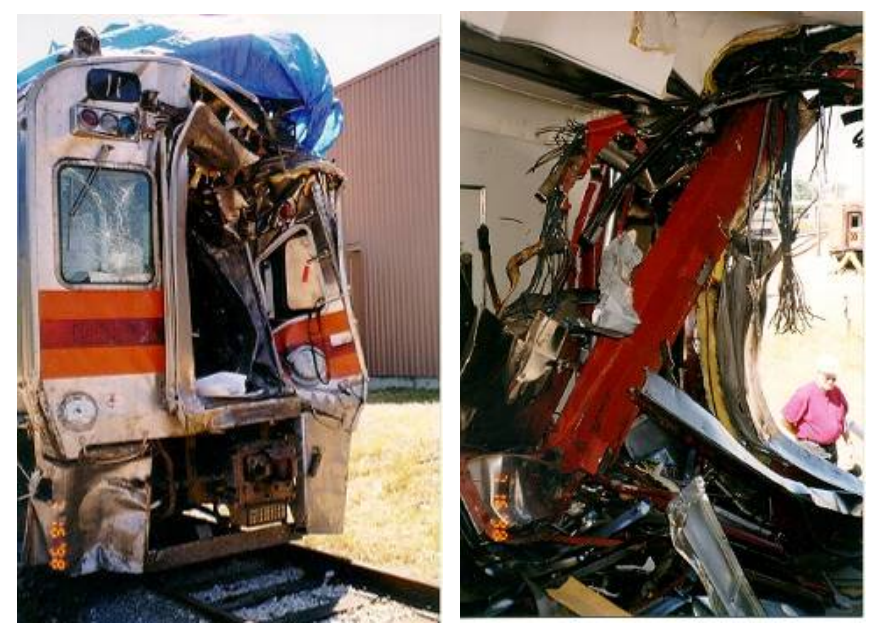

Figure 1. Cab car involved in the grade crossing collision in Portage, Indiana

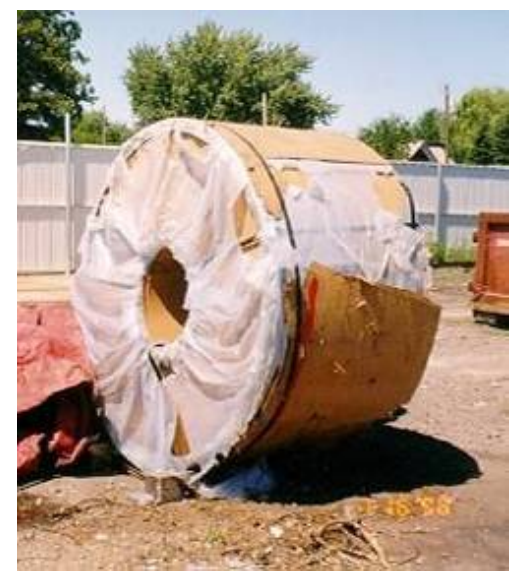

Figure 2. Steel coil involved in the grade crossing collision in Portage, Indiana

Collision in Selma, North Carolina On May 16, 1994, an overhanging intermodal trailer on the northbound freight train was obstructing the way of the southbound intercity passenger train [2]. The forward trailer of the $51^{\text {st }}$ car was overhanging the southbound track and engaged the lead locomotive of the passenger train. At the onset of contact, the trailer was above the deck and offset outside the collision posts of the passenger train lead locomotive. One operator was killed and another operator was injured during the collision.

This collision is significant because the trailer impacted the superstructure of the car, not the underframe.

\section{PASSENGER CRASHWORTHINESS PROGRAM}

An ongoing objective has been to evaluate the existing passenger car designs and offer potential improvements. There have been three full-scale dynamic tests with a grade-crossing scenario. Two tests focused on the corner post, testing a generalized 1990s design and an improved SOA design. This test focused on the collision post of a SOA collision post.

State-of-the-Art End Frame The SOA end frame is a specific end frame prototype design developed for this series of tests. There are several improvements over the 1990s design. These improvements are intended to absorb more energy during a collision and provide a survivable space for the operator and passengers. The SOA design, shown in Figure 3, includes more substantial collision and corner posts. The connections between the corner and collision posts and the AT beam along the top of the end frame, and the buffer beam along the bottom of the end frame, are made stronger by running the posts through the entire beams. Shelves and bulkhead sheets connect the collision and corner posts, allowing some load to be shared between the two posts. The end frame is better supported by a continuous side sill and robust roof rails $[4,5]$. 


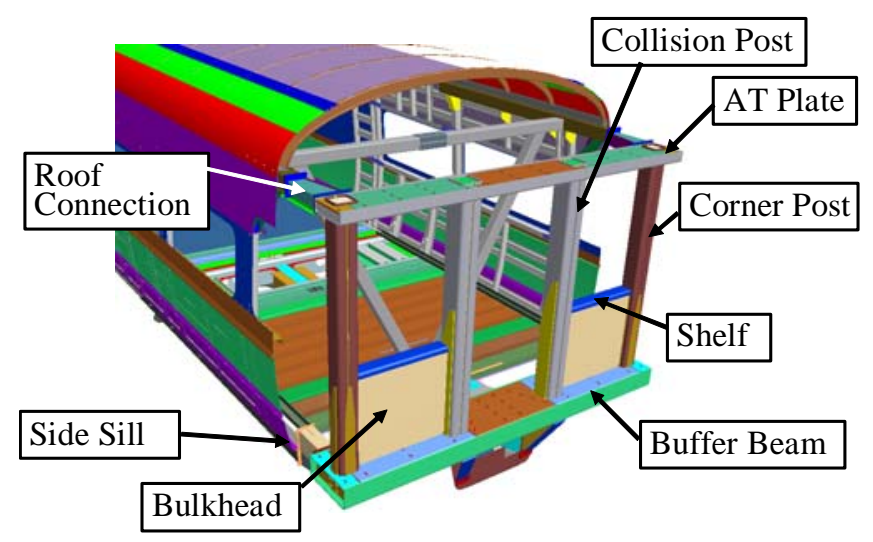

Figure 3. Schematic of the state-of-the-art end frame

Test Program Table 1 shows the full-scale tests that have been performed as part of the grade-crossing scenario research. Two dynamic tests were performed on the corner posts. One test was performed on a 1990s design, which was designed to be a typical end frame structure of the 1990s. The second dynamic corner post test was conducted on a SOA design.

This paper discusses the dynamic test of the collision post of the SOA end frame. Two more quasi-static tests are planned on the state-of-the-art end frame, one on the collision post and one on the corner port. These tests are planned for the summer of 2008.

Table 1. End Frame Tests

\begin{tabular}{|l|l|l|}
\hline Test Type & Post & Design \\
\hline Dynamic & Corner & 1990 s \\
\hline Dynamic & Corner & SOA \\
\hline Dynamic & Collision & SOA \\
\hline Quasi-static & Collision & SOA \\
\hline Quasi-static & Corner & SOA \\
\hline
\end{tabular}

Corner Post Tests There have been two previous dynamic tests, both on corner posts. The first test was conducted on the corner post of a 1990s design end frame. In this test, the car had an initial velocity of approximately 14 mph and collided with a 6-foot diameter, 40-kip steel coil. The coil was originally mounted on a frangible wooden table. The center of the coil hit the corner post at a height of 30 inches above the top of the finished floor. During this test the connection between the corner post and the AT beam failed.

A second dynamic test was conducted on the corner post of a SOA end frame. The same test setup was used as in the 1990s design test. During this test, the corner post and attachments did not fail. After impact, the coil rolled under the train, tipping the car. The car did not derail, but the unexpected tipping was a cause of concern for future tests [6].

Proposed Rule At the time of the writing of this paper, FRA has released an NPRM. The proposed rule increases the structural strength requirements for the front end of cab cars and MU locomotives [1]. The improvements include the addition of deformation and energy absorption requirements specified in the revised American Public Transportation Association standards for front end collision posts and corner posts [7]. In addition, the NPRM requires car builders to use either a dynamic or quasi-static test to determine if their design absorbs the appropriate amount of energy within the desired distance. By allowing an alternative dynamic scenario, nontraditional designs may be tested for an equal amount of energy absorption.

In the NPRM dynamic collision scenario for a collision post, the end frame must absorb 135,000 ft-lb of energy during the collision. In the specific scenario, a cab car traveling at 21 mph hits a proxy object. The proxy object has a diameter of 48 inches and a height of 36 inches. The cylinder contacts the collision post at 30 inches above the height of the finished cab floor. The proxy object must have a minimum weight of 10,000 $\mathrm{lb}$ and the cab car must have a minimum weight of $100,000 \mathrm{lb}$.

When subjected to this collision scenario, the collision post must not crush more than 10 inches longitudinally into the passenger compartment. Also, there must be no complete separation of the posts from their attachments.

\section{COLLISION POST TEST DESIGN}

Several alternative methods for an equivalent dynamic test were considered, including running the car into a stationary coil shape, mounting the coil shape onto a pendulum, and hitting the collision post. After considering the test cost, safety and repeatability, it was decided to build the coil shape onto an existing cart. A rigid cart design allows the same cart to be used for multiple tests, on either the corner or collision post. The test facility is familiar with running dynamic tests and can reliably set the speed of the cart. Nothing except the test article and the strain gages were destroyed with this test set up. The strain gages were included in this test for research purposes and are not necessary to pass the NPRM dynamic test. Figure 4 shows a schematic of the test layout. The cab car is on the left of the figure and the cart is on the right.

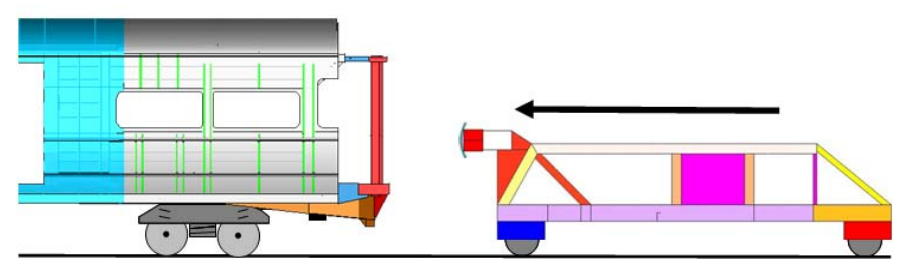

Figure 4. Schematic of the dynamic test layout

The energy absorbed during the test, the final energy subtracted from the initial energy, is the key factor in showing the post's crashworthiness. For test control and safety reasons, the cart, not the cab car, was given the initial velocity as described in the NPRM. The initial velocity of the cart was adjusted for the lower weight of the cab car and the higher 
weight of the cart. The target initial velocity, $19 \mathrm{mph}$, ensured that the end frame would absorb a minimum of 135,000 ft-lb of energy during the collision. The actual test velocity was measured at $18.7 \mathrm{mph}$, which also met the energy requirements.

Cart Design A proxy object cart mounted with a coil shape was developed as an alternative to the steel coil on a frangible table.

According to the NPRM, the proxy object must have the following characteristics:

- Diameter $=48$ "

- Length $=36$ "

- Weight $>=10$ kips

- Longitudinal axis = 30" above floor

- Withstand $21 \mathrm{mph}$ impact without deformation

A retired ballast vibrator was used to fabricate the proxy object cart. The center section of the vibrator was removed and the end sections were welded to each other to reduce the weight of the cart. The cab was removed and the cart was stripped of components that were considered nonstructural. Additional struts were added to ensure that the cart would act as much like a rigid body as possible. A coupler was also added to the cart frame to facilitate movement of the cart.

A coil shape with a 48-inch diameter and 36-inch width was mounted on a box beam on the leading end of the cart, at the required height of 30 inches above the finished floor of the cab car. The beam supporting the coil shape was designed so that the coil shape could be moved horizontally in order to impact the corner post, if necessary. The coil shape could also be moved vertically a few inches in either direction for proper location for impact. The finished cart, shown in Figure 5, weighed approximately 14 kips.

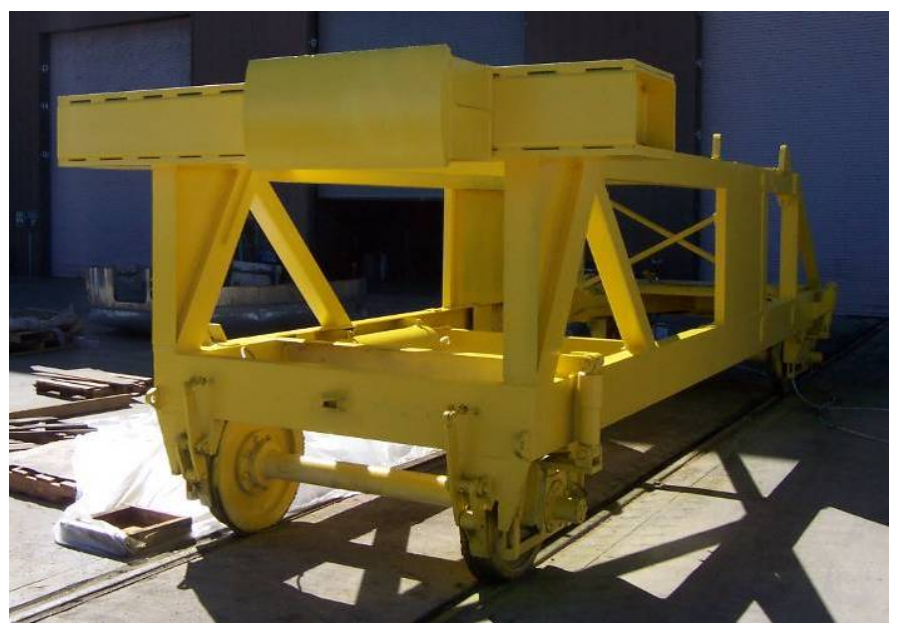

Figure 5: Finished Proxy Object Cart

A finite element (FE) model of the cart was generated in HyperMesh 8.0, as shown in Figure 6. Finite element analyses (FEA) were conducted in ABAQUS 6.7-1 to determine the mode of deformation of the cart, to confirm that the cart could withstand an impact with the cab car, without permanent deformation [5]. Analyses were also conducted to determine the gross motions of the cart and the cab car as a result of the impact, and particularly to ensure that the impact with the cart would not cause the cab car to derail.

In the cart analyses, the collision post was modeled as a rigid body attached to a spring whose force-displacement behavior was derived from the FEA of the cab car and steel coil. Most of the cart components consisted of steel with a strength of $38 \mathrm{ksi}$. However, the coil shape was made of $50 \mathrm{ksi}-$ strength steel.

The cart impacted with the rigid collision post body at a speed of $21 \mathrm{mph}$, travelled approximately 8 inches, and then rebounded backwards with a yaw of the rear of the vehicle of less than $2^{\circ}$. Figure 7 shows the stresses in the cart after impact. As shown in the figure, the maximum stresses found in the coil shape are just at their 50-ksi yield strength, and these maximum stresses are only at the location of contact with the rigid body. The stresses in the rest of the cart are well below their 39-ksi yield strength.

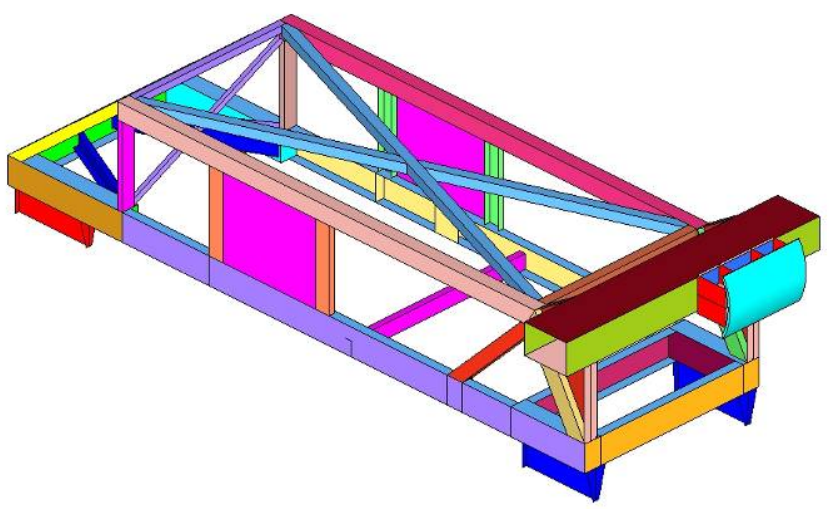

Figure 6: Finite Element Model of the Proxy Object Cart

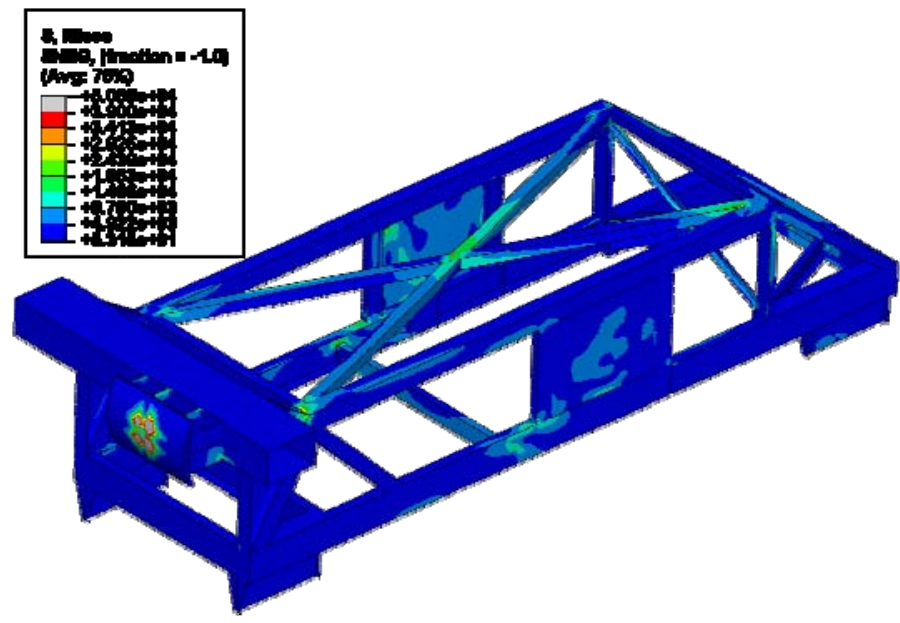

Figure 7: Von Mises Stresses of the Cart 
A final FEA was conducted with the cab car and the cart [5]. In this analysis, the cart was modeled as rigid and impacted the collision post of the cab car at $21 \mathrm{mph}$. The results of this analysis compared very favorably with the results of the 10-kip coil impacting the cab car [5]. The analyses resulted in similar yaw-time histories between the coil and the cart, similar collision post displacements, and similar energies absorbed in the collision. The FEAs conducted with the proxy object cart confirmed that the dynamic collision would not cause the cab car to derail and would, at worst, only cause the proxy object cart to derail.

Retrofit The test article was a Budd M1 car, donated to FRA from the Long Island Rail Road. The front end of the car was cut off and replaced with a SOA end frame. The end frame design was the same design used in the previous corner post tests [8]. The original SOA end frame was designed to be retrofit onto a Budd Pioneer car. Since only a Budd M1 car was available for these tests, the attachments had to be altered to attach to the Budd M1 car. The connection and reinforcement parts were checked by FEAs to ensure they would not deform plastically during the test [5].

Collision Dynamics Model A collision dynamics model was developed prior to the test to predict the gross motions of the colliding vehicles, predict the crush of the end frame and to determine the instrumentation necessary to obtain critical measurements. This type of model was used for the dynamic corner posts test [9].

The model was developed using ADAMS, a commercial software package. The lumped-mass model of the test scenario includes a spring-mass representation of each vehicle. The inertial properties are defined for each mass. As the cart impacts, non-linear springs are used to model the force-crush behavior of the end frame. The passenger rail car has linear spring connections to two rigid bodies representing the truckto-carbody suspension. Figure 8 shows a schematic of the lumped-mass model.

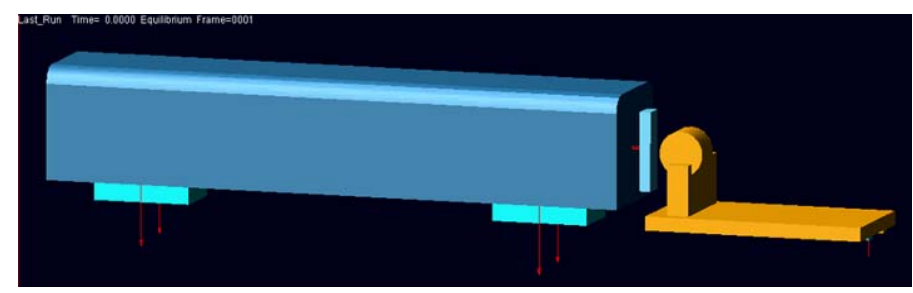

Figure 8: Collision Dynamics Model of the Impact Scenario

Figure 9 shows force-crush characteristic derived from the pre-test FEAs conducted on the end frame [5].

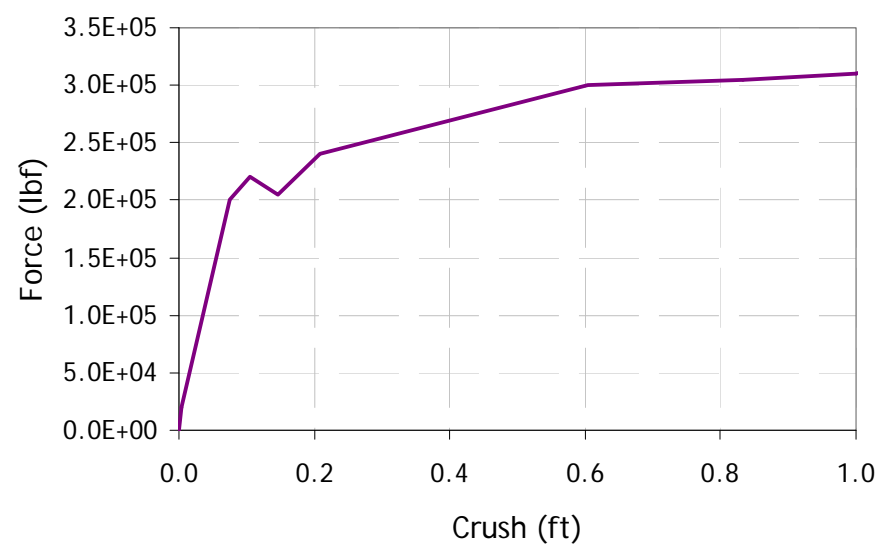

Figure 9: Idealized Force-Crush Behavior of State-of-theart Collision Post

Figure 10 shows the pre-test predictions for gross motions of the car and the cart. The passenger car is initially at rest. The simulation is exercised at a target speed of $19 \mathrm{mph}$ for the cart. The end frame crushes by approximately 6 inches. The cart rebounds off the car at approximately $1.5 \mathrm{mph}$ and the car moves away at approximately $3 \mathrm{mph}$. The rebound speeds are low and manageable for the test facility.

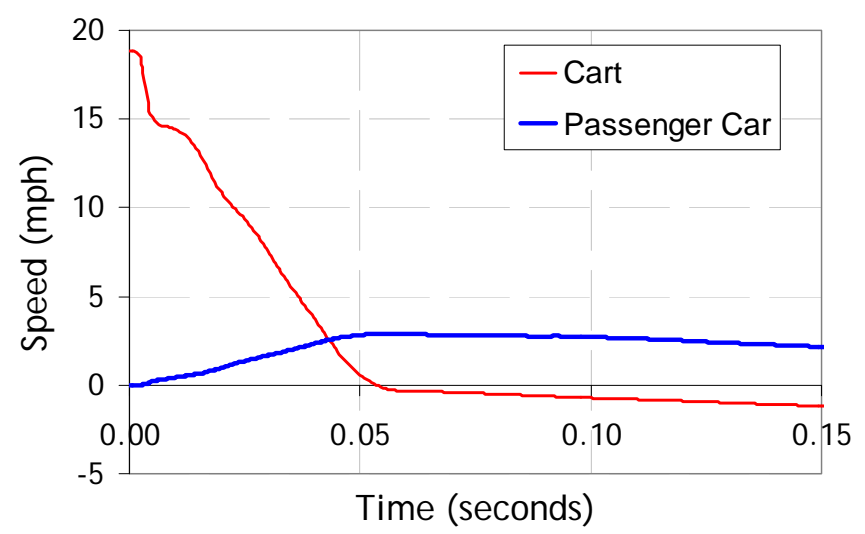

\section{Figure 10: Pre-test Prediction of Longitudinal Gross Motions of the Colliding Vehicles}

The collision dynamics model showed that the cart would pitch upward and yaw after it impacted the end frame. The vertical loads, combined with the yaw motions from the offset load, would likely cause the cart to derail during the test.

\section{TEST RESULTS}

The actual test speed was $18.7 \mathrm{mph}$. The collision post indented approximately 7.375 inches, meeting the requirement that there be less than 10 inches of crush. The end frame exceeded the energy absorption requirement by absorbing 138,000 ft-lb of energy. Figure 11 shows still frames of the test taken from the high speed video. The top photo shows the cart at the first impact. The second photo shows the cart and 
collision post at the maximum amount of collision post deflection. The third photo shows the cart and end frame at the end of the test, after some elastic energy has been recovered form the collision post. The cab car moved back approximately 6 feet after the test and stopped. The cart lifted vertically and moved laterally on recoil. When the cart came down after impact, the wheels missed the track and the cart derailed. Since the cart came down off the tracks, it stopped and did not move backwards.
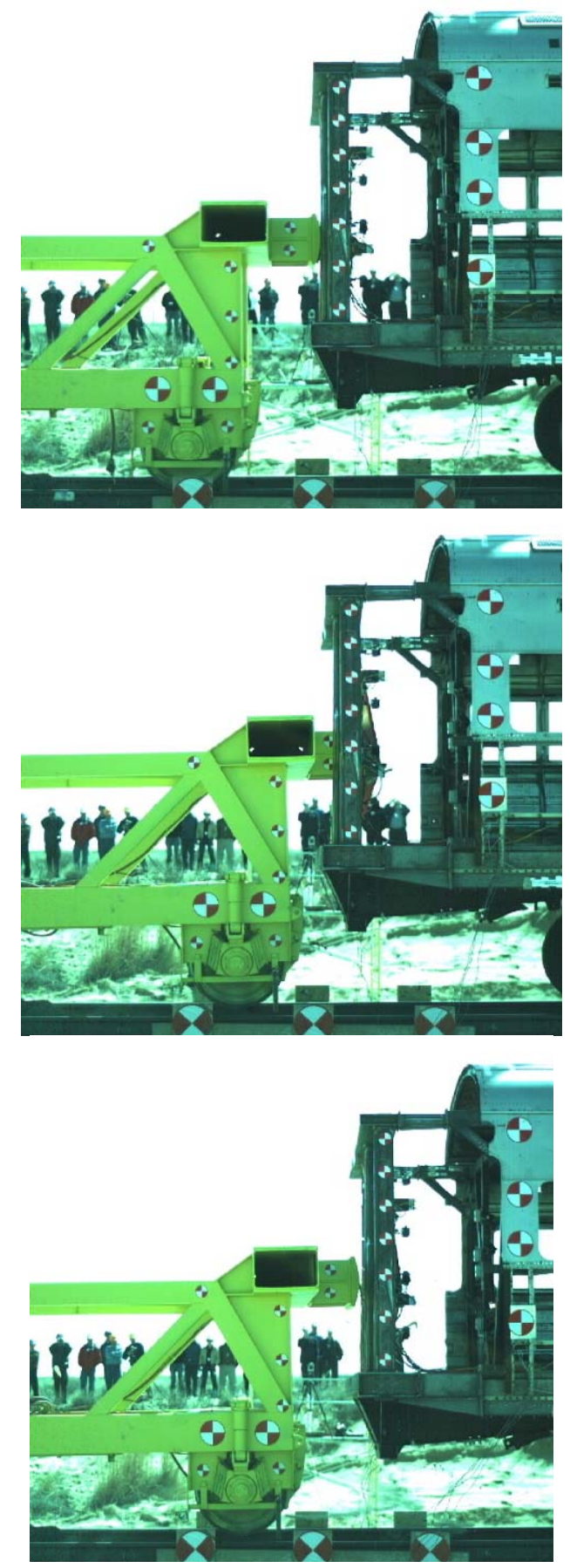

Figure 11. Still photographs from high-speed video
End Frame Performance Figure 12 shows the end frame after the test. The impacting object pushed in the collision post, lateral shelf and bulkhead sheet. The collision post partly separated from the buffer beam, as did the bulkhead sheet. The shelf separated from the collision post and the corner post. The AT beam, which runs laterally at the top of the end frame, bent down at the connection to the collision post. The welds between the collision post and the AT beam did not crack or fail.

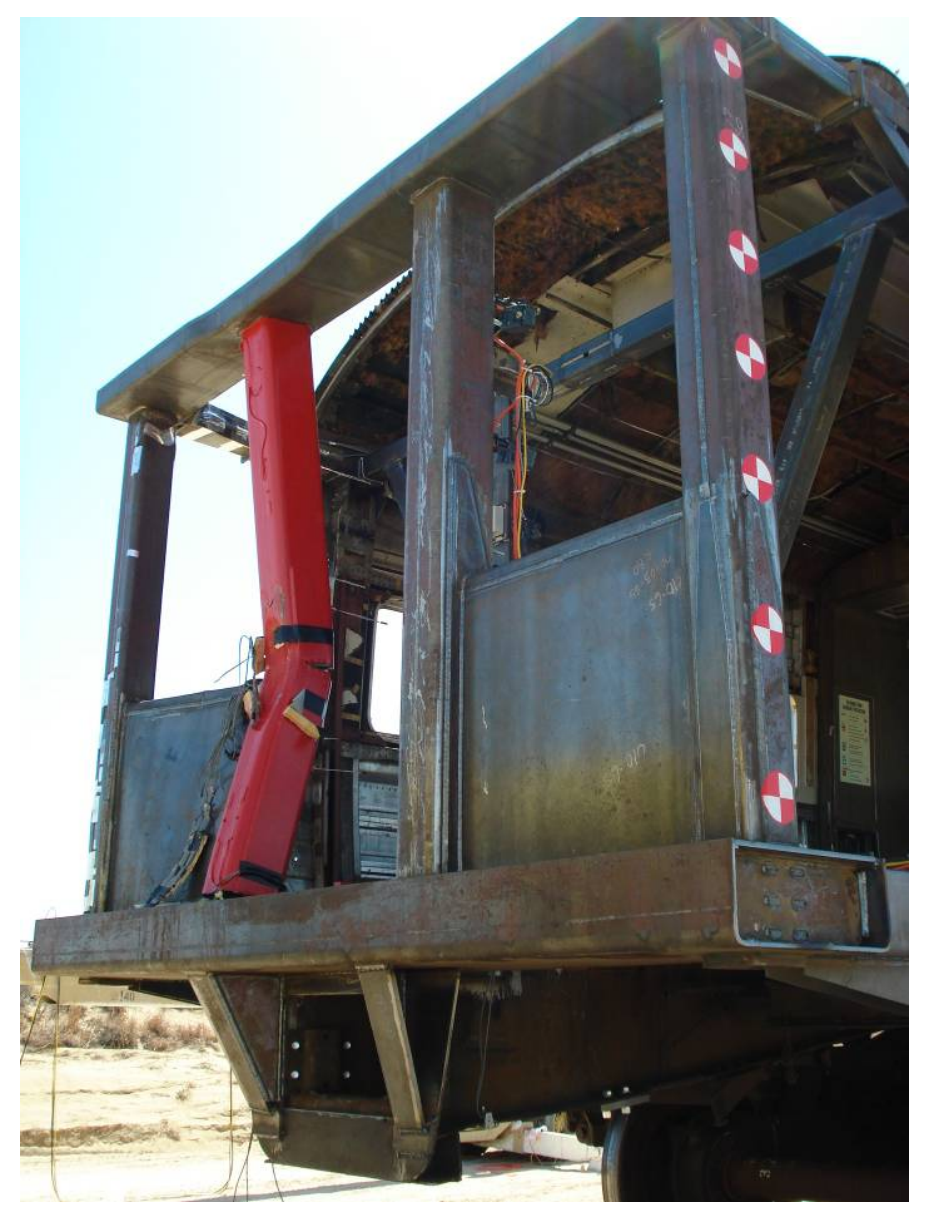

Figure 12. A photograph of the end frame after the test

Figure 13 shows the back of the collision post and the connection to the shelf before the test. The tab extends from the shelf and is welded to the back of the collision post. There is a gap between the side of the post and the shelf. The post is a box cross section formed by two U-sections welded together at the front and back of the post. There are several internal gussets in the horizontal plane. 


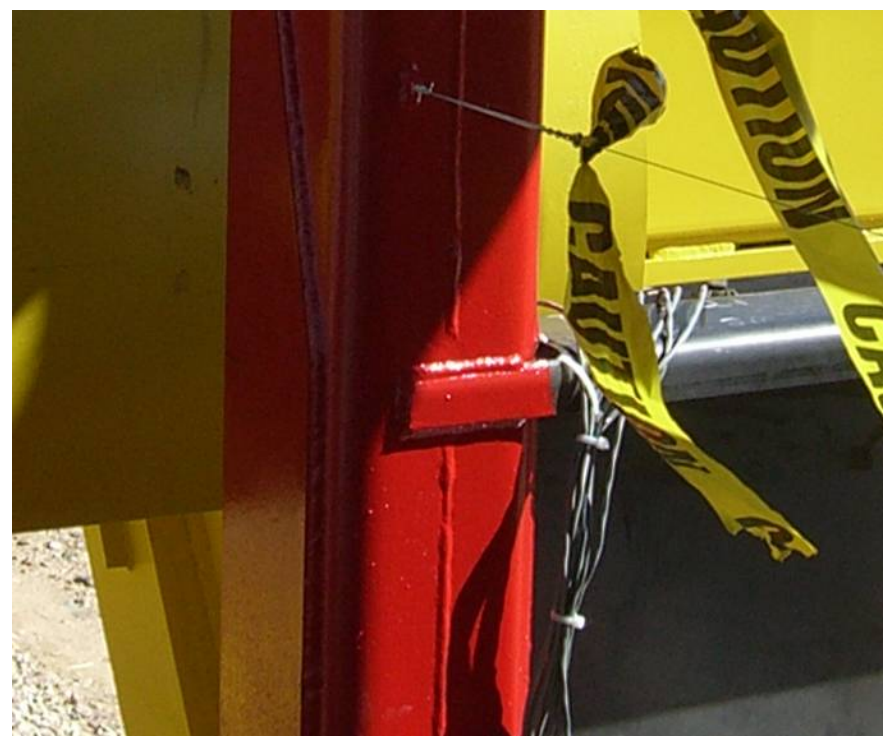

Figure 13. The back of the collision post, at the connection to the shelf, pre-test

Figure 14 shows the back of the collision post and the connection to the shaft, after the test. The post fractured along the backside and almost entirely along the sides of the post. The front of the post did not fracture. The shelf fractured at the location of the gap between the collision post and the shelf. There are external gusset plates along both the sides of the posts. The plates fractured also.

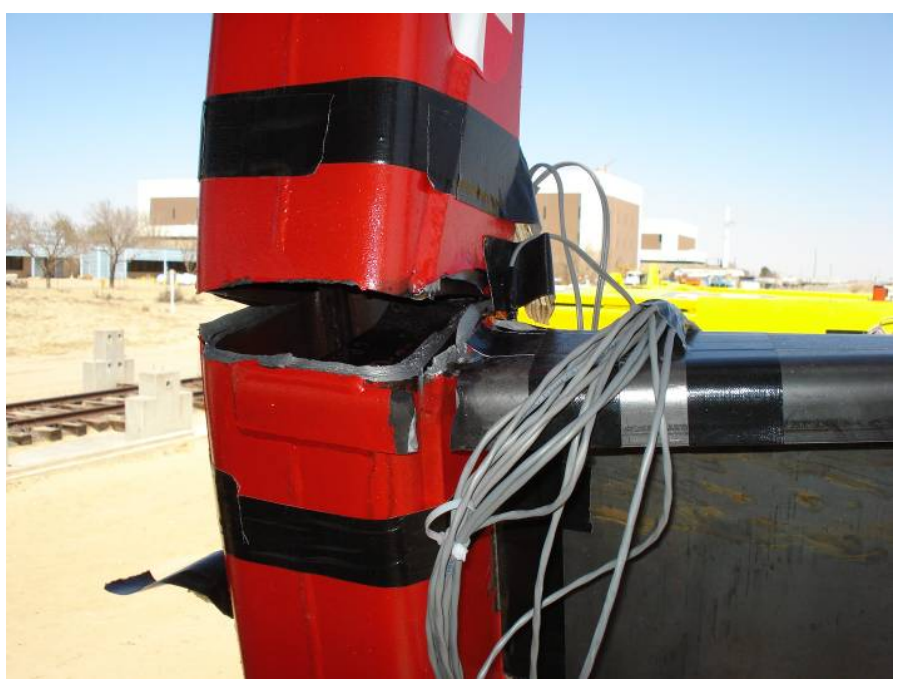

Figure 14. The back of the collision post, at the connection to the shelf, post-test (bottom)

Figure 15 shows the connection of the shelf to the back of the corner post before the test. This connection is similar to the connection between the shelf and the collision post. There is a gap between the shelf and the corner of the corner post.

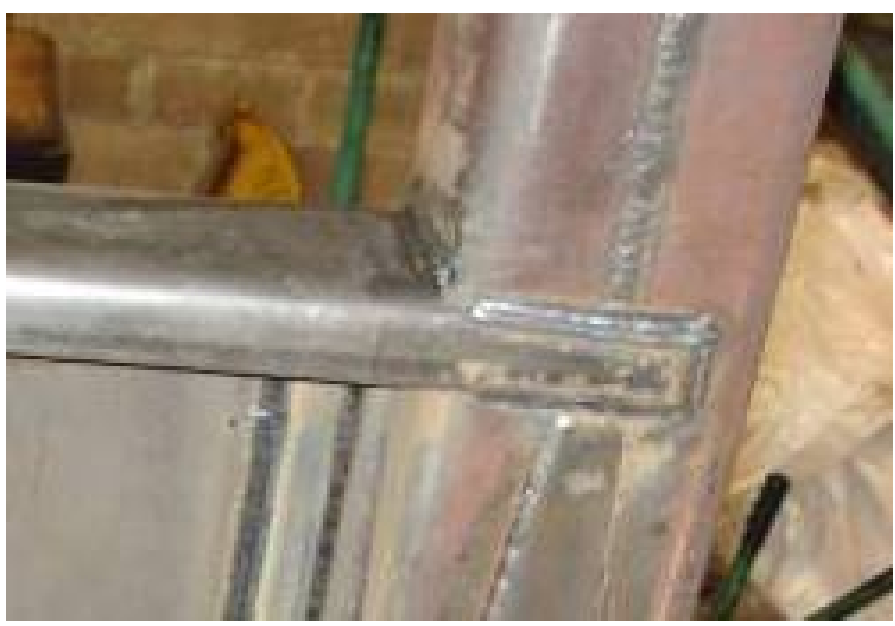

Figure 15. The shelf connection to the corner post, pre-test

Figure 16 shows the connection between the corner post and the shelf, after the test. During the test, the shelf was pushed in longitudinally toward the center of the train and outward laterally toward the corner post. The corner post has been pushed in and deformed plastically at the shelf. Also, the tab of the shelf fractured off.

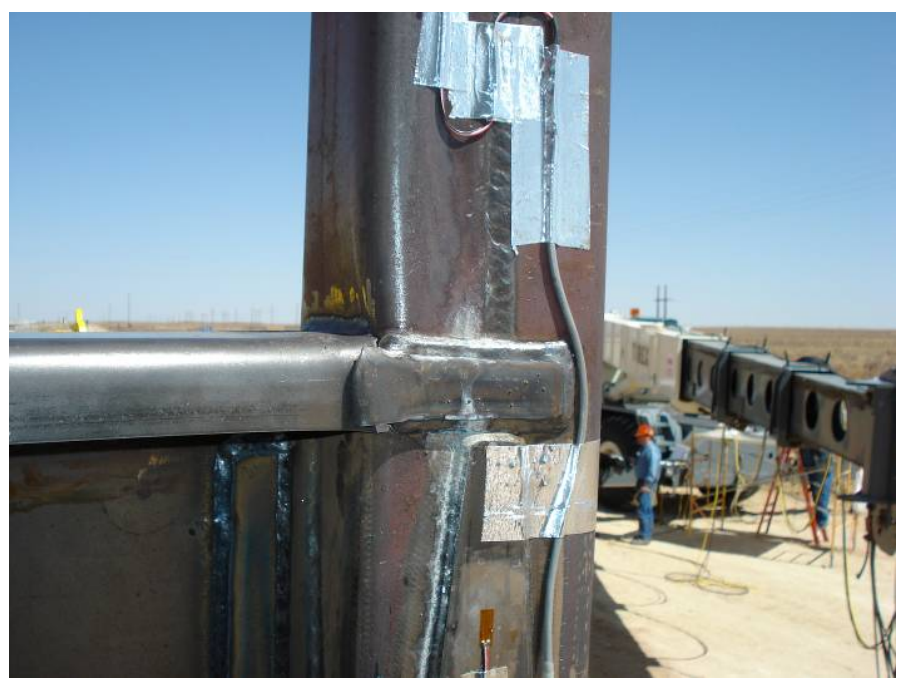

Figure 16. The connection of the shelf to the corner post, post-test

The collision post fractured at the connection to the buffer beam. Figure 17 shows the fracture. The bulkhead sheet tore from the buffer beam at the corner. The crack on the left side of the post (as seen in the picture) extends behind the bulkhead sheet. On the right side of the post, the crack extends almost down the entire side. The buffer beam was distorted and the top plate of the buffer beam pushed down at the location of the collision post and the bulkhead sheet. 


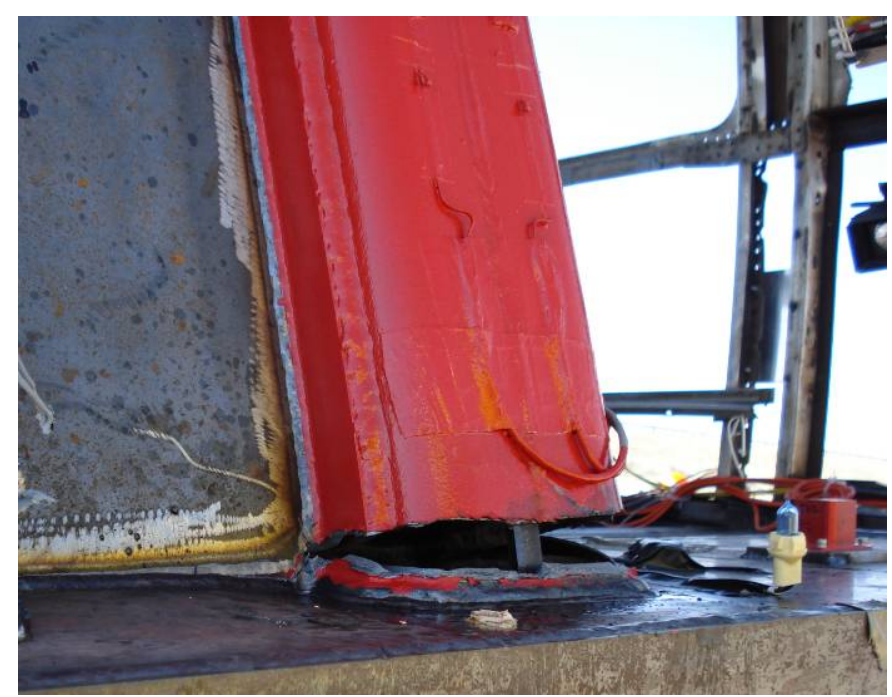

Figure 17. Fracture at the connection between the collision post and the buffer beam

Gross Motions Figure 18 shows the velocity time history for the cab car and the cart. The test data time histories are calculated from accelerometers mounted on both the cab car and the cart. The pretest predictions come from the collision dynamics model. The cab car is initially standing and travels at approximately $3 \mathrm{mph}$ after the impact. This matches the pretest collision dynamics prediction. The cart impacts the car at 18.7 mph, rebounds off of the passenger car, derails, and stops. The pretest prediction estimated that is would take 0.05 second for the cart to nearly stop. The test data shows that the cart takes 0.12 second to stop. The energy absorbed by the end frame during the test was 138,000 ft-lb.

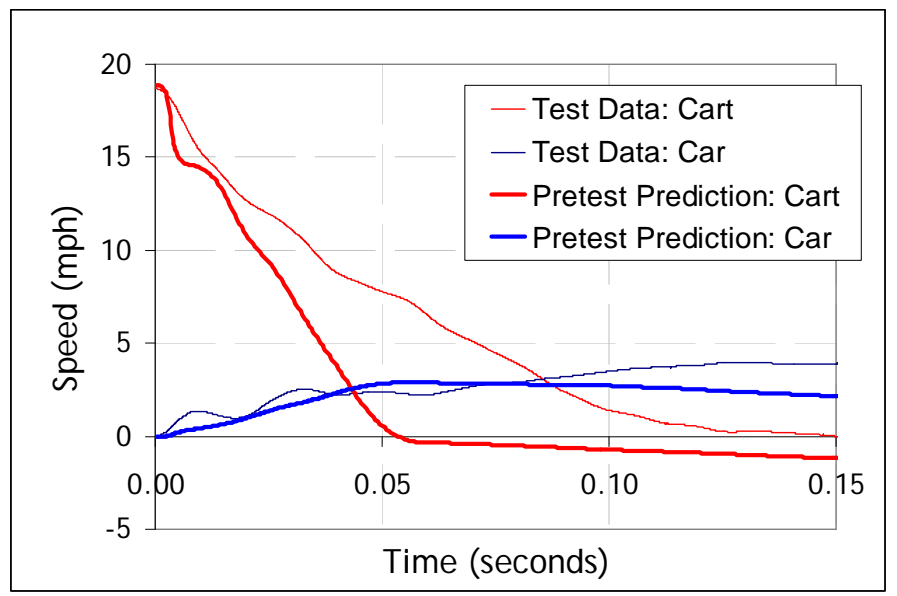

Figure 18. Accelerometer data, velocity vs. time for the cart and cab car

Cart Performance The most important requirements for the cart were that it not deform or absorb any energy during the collision. A rigid cart ensured that the collision post and end frame would absorb the required collision energy. Figure 19 shows the impactor after the test. The cart performed as expected during the test and did not deform. After the test, the cart derailed as expected, due to the off-center load. The cart remained upright. The derailment did not damage the cart.

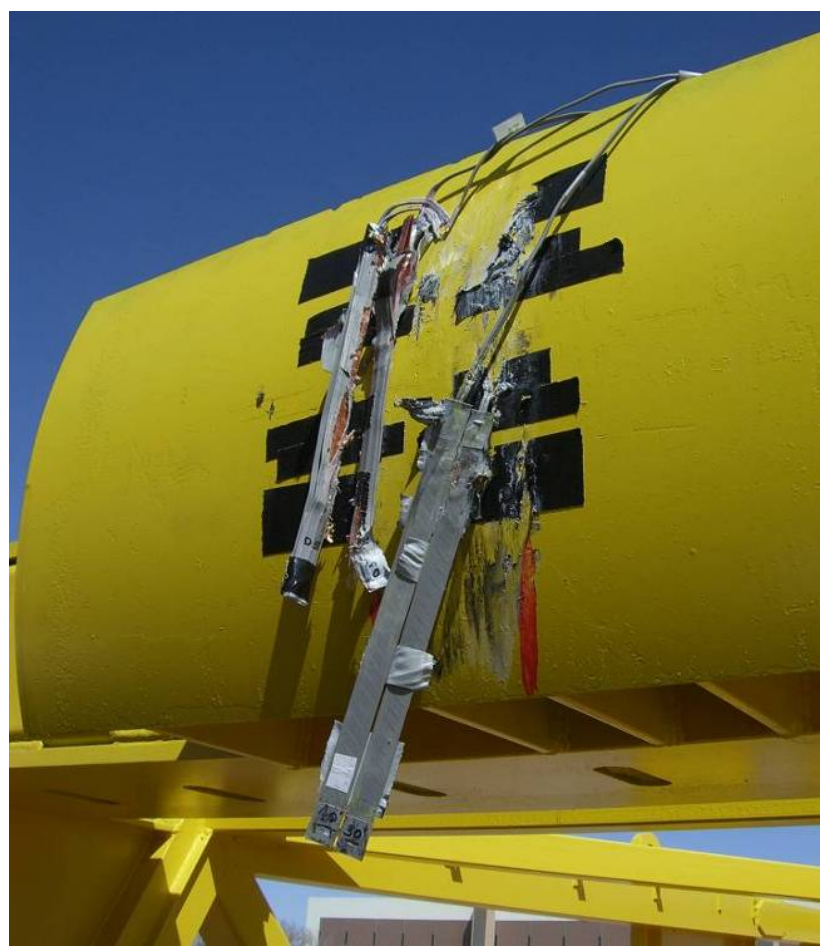

Figure 19. Cart impactor, post-test

\section{SUMMARY}

A dynamic test was performed on the collision post of a SOA end frame in support of a notice of proposed rulemaking for passenger cars. During the test, a cart weighed 14,000 lb with a cylinder-shaped impactor on the end, traveled at 18.7 mph and hit the collision post of a standing cab car weighing $70,000 \mathrm{lb}$. The cart did not deform plastically during the test. The cab car end frame absorbed 138,000 ft-lb of energy, exceeding the requirement of $135,000 \mathrm{ft}-\mathrm{lb}$ of energy. The collision post plastically deformed 7.375 inches, meeting the requirement of less than 10 inches of deformation.

This collision scenario for this test was modified from the NPRM scenario. These changes ensured a safe, economical, and repeatable test.

\section{PLANNED WORK}

The series of test includes two quasi-static tests, also modeled after the requirements in the notice of proposed rulemaking. A quasi-static test of a SOA collision post occurred on June 25, 2008 and the quasi-static test of a SOA corner post will occur in the late summer of 2008. The knowledge gained 
from these test will be presented to the industry working groups in support of the notice of proposed rulemaking.

\section{ACKNOWLEDGMENTS}

The research described in this paper was performed as part of the Equipment Safety Research Program sponsored by the Office of Research \& Development of FRA. Eloy Martinez, Program Manager, led this effort. Luis Maal, FRA Resident Manager at the Transportation Technology Center, managed the full-scale test effort.

Richard Stringfellow and Chris Paetsch, of TIAX, ran a pretest finite element model and monitored the purchase and manufacturing of the parts of the end frame, under contract to the Volpe Center.

Gabriel Amar of Taylor, Raynauld, Amar and Associates provided the cut drawings and the SOA design drawings.

Mark White of Transportation Technology Center, Inc. (TTCI), led the TTCI team that implemented the test. Tom Roderick of TTCI, led the fabrication of the end frames and retrofit onto the existing cars.

Long Island Railroad donated the Budd M1 car used in this test.

\section{APPENDIX}

As part of the test design, instrumentation layouts are specified. The instrumentation includes accelerometers, strong potentiometers, strain gages, real-time digital video and uncompressed digital video. The instrumentation specified here is not necessary for running a dynamic test. The instrumentation allows for further study of rail crashworthiness. The test article could demonstrate that it met the requirements without instrumentation.

Accelerometers on the cart and cab car are intended to capture the gross motions of the cart and cab car. The ranges of the accelerometer were chosen based on the acceleration levels predicted in the collision dynamics model. Table A1 describes the accelerometer directions and prescribed ranges.

Table A1. Accelerometer data channels and ranges

\begin{tabular}{|l|l|l|l|}
\hline Car & Direction & Range (g) & \# of channels \\
\hline Cab Car & Longitudinal & 10 & 4 \\
\hline & Vertical & 10 & 4 \\
\hline & Lateral & 5 & 2 \\
\hline Cart & Longitudinal & 100 & 5 \\
\hline & Vertical & 20 & 6 \\
\hline & Lateral & 5 & 4 \\
\hline \multicolumn{2}{|c|}{ Total \# of Data Channels } & 25 \\
\hline
\end{tabular}
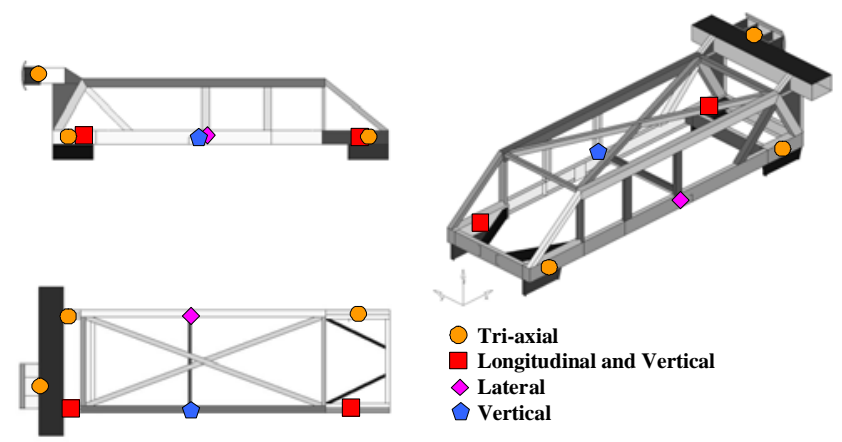

Figure A1. Schematic of the accelerometers on the cart

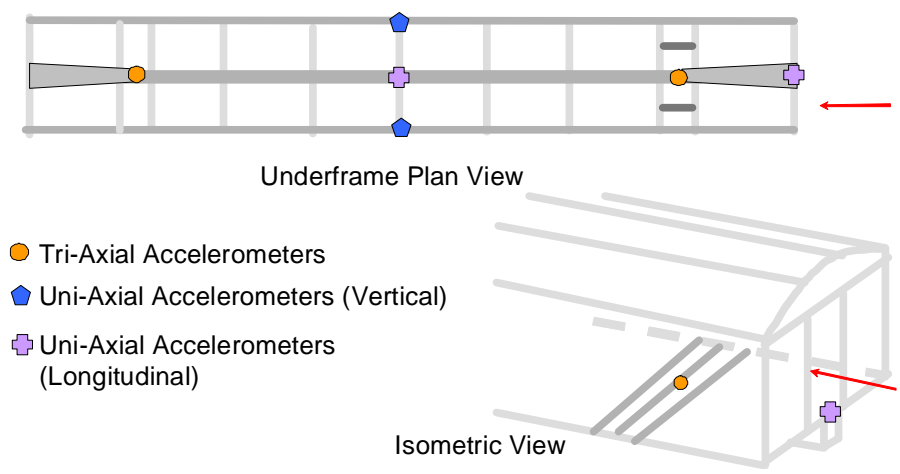

Figure A2. Schematic of the accelerometers on the cab car

The strain gages on the cab car capture the timing of the stress wave through the end frame and the cart. The location of the strain gages were chosen based on results from the finite element model. The strain gages are placed on locations where plastic deformation is not anticipated. The ranges for the strain gages were chosen based on the predicted strain values from the finite element model.

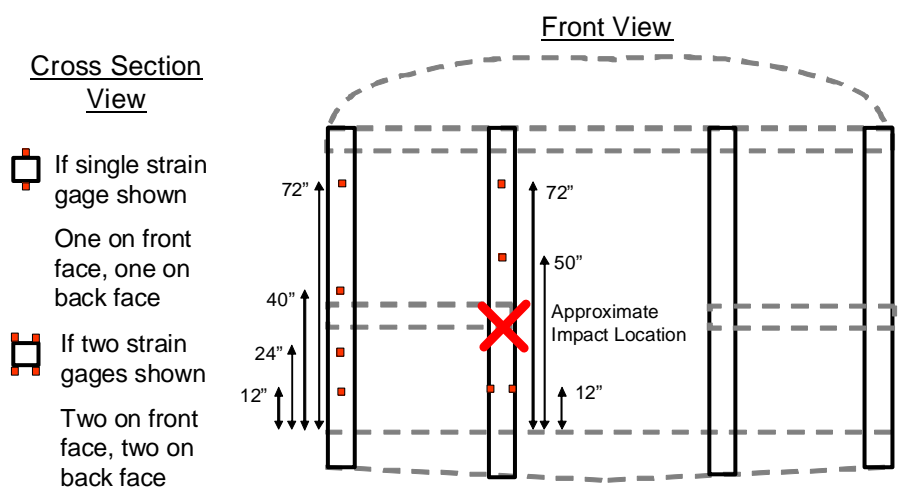

Figure A3. Schematic of strain gages on the cab car collision and corner posts 


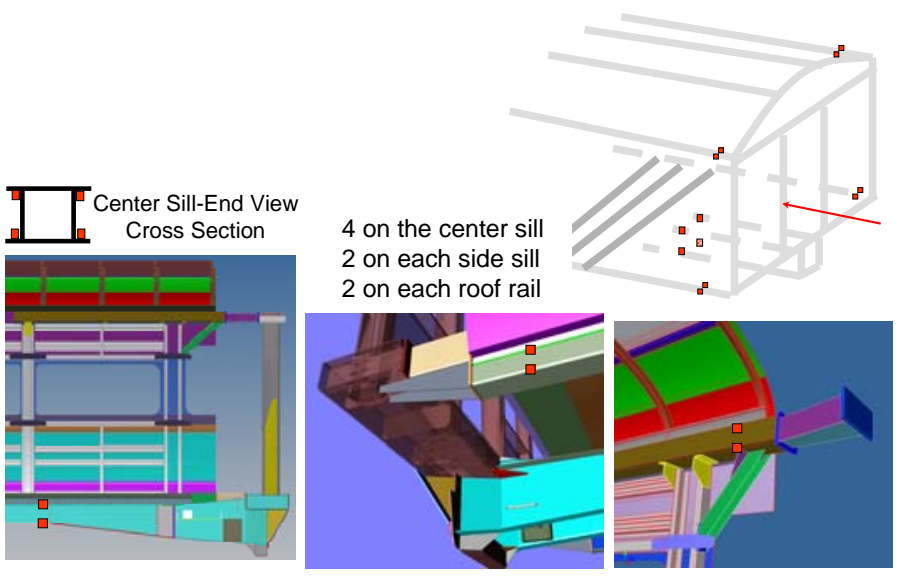

Figure A4. Schematic of strain gage placement on the cab car longitudinal members

Four string potentiometers were placed on the collision post in the longitudinal direction. These instruments capture the mode of deformation of the collision post during the test. One string potentiometer was placed at each $1 / 3$ point and two were placed near the midpoint of the collision post.

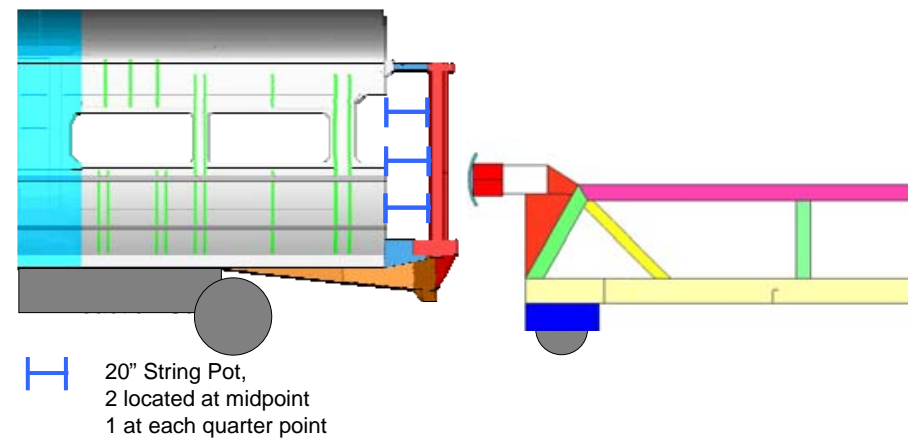

Figure A5. Schematic of the string potentiometers on the collision post

Both real-time and high speed, uncompressed digital video are use to capture the gross motions and modes of deformation during the test. The locations of the external cameras are shown in Figure A6. There were also video cameras with the high frame rate set up in the cab car. One camera focused on the connection of the collision post to the AT beam. The other camera focused on the connection of the collision post to the buffer beam. The rate of the high speed cameras was 1000 frames per second. The frame rate of the real-time cameras was 30 frames per second.

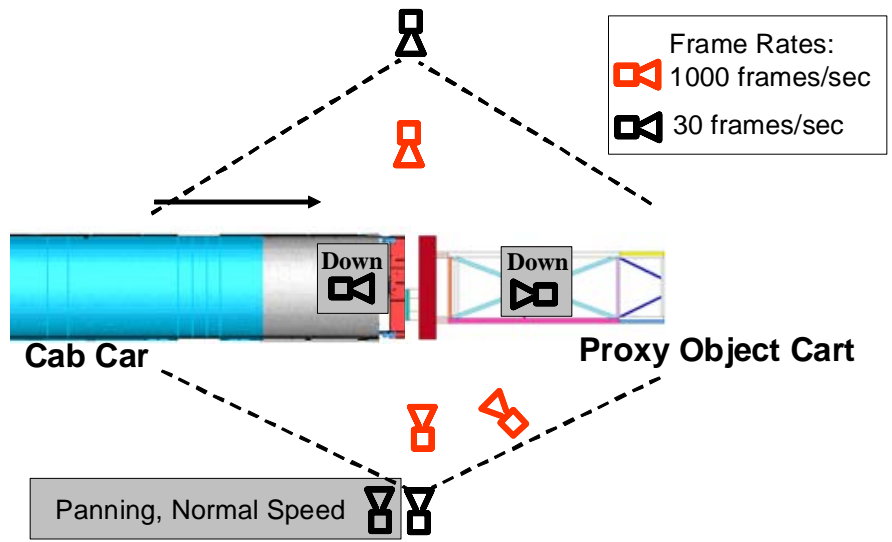

Figure A6. Schematic of the placement of external video

\section{REFERENCES}

[1] 49 CFR Part 238, Notice of Proposed Rulemaking, Passenger Equipment Safety Standards; Front End Strength of Cab Cars and Multiple Unit Locomotives, Department of Transportation, Federal Railroad Administration, Washington, D.C.

[2] Tyrell, D., "Rail Passenger Equipment Accidents and the Evaluation of Crashworthiness Strategies,” Journal of Rail and Rapid Transit, Proceedings Part F, Institute of Mechanical Engineers, August 2002.

[3] National Transportation Research Board, "Collision of Northern Indiana Commuter Transportation District Train 102 with a Tractor-Trailer, Portage, Indiana, June 18, 1998” 07/26/1999, RAR-99-03.

[4] Mayville, R., Stringfellow, R., and Martinez, E., "Development of Conventional Passenger Cab Car End Structure Designs for Full Scale Testing.” U.S. Department of Transportation, DOT/FRA/ORD-06/20, December 2006.

[5] Stringfellow, R., Paetsch, C., and Amar, G., "Development and Fabrication of State-of-the-Art End Structures for Budd M1 Cars,” American Society of Mechanical Engineers, Paper No. RTDF2008-74033, September, 2008.

[6] Tyrell, D., Martinez, E., Jacobsen, K., and Perlman, A. B., "Passenger Cab Car Grade Crossing Impact Test Report." U.S. Department of Transportation, DOT/FRA/ORD07/24, October 2007. 
[7] APTA SS-C\&S-034-99, Revision 2, Standard for the Design and Construction of Passenger Railroad Rolling Stock, The American Public Transportation Association, Washington, D.C.

[8] Mayville, R., Stringfellow, R., Johnson, K., Tyrell, D., "Rail Vehicle Car Cab Collision and Corner Post Designs According to APTA S-034 Requirements," American Society of Mechanical Engineers, Paper No. MECE200344114, November 2003.

[9] Jacobsen, K., Tyrell, D., Perlman, A.B., "Rail-Car Impact Tests with Steel Coil: Collision Dynamics," American Society of Mechanical Engineers, Paper No. JRC20031655, April 2003. 